\title{
Neuroleptic Malignant Syndrome or Catatonia? A Case Report
}

\author{
Sebastian Rodriguez ${ }^{1 *}$, Keith A. Dufendach², Robert M. Weinreib3 \\ 1 University of California San Francisco, San Francisco, USA \\ 2 University of Pittsburgh Medical Center, University of Pittsburgh, Pittsburgh, USA \\ 3 University of Pennsylvania Health System, Perelman School of Medicine, University of Pennsylvania, USA
}

\begin{abstract}
Introduction: A review of the literature has shown that there are many similarities in the presentation of neuroleptic malignant syndrome (NMS) and catatonia. Attempts to reconcile the differences have been made by suggesting that NMS and catatonia may represent different presentations of the same illness or that they lie within the same spectrum of a poorly understood clinical syndrome. The described case is of a patient who presented with NMS and catatonia which was difficult to diagnose, but which responded to treatment with intravenous diazepam. Case presentation: The case concerns a 22-year-old male admitted for pulmonary hypertension to an intensive care unit (ICU). Three days following admission, he developed a high fever that did not respond to antibiotics. The patient then developed rigidity, nocturnal agitation, decreased responsiveness, and somnolence. Without the use of bromocriptine (Novartis, Basel, Switzerland) or dantrolene (Par Pharmaceuticals, Chestnut Ridge, USA) discontinuation of neuroleptics combined with intravenous diazepam (Pfizer, NY, USA) led to a very rapid response and marked improvement in the case. Conclusions: Early recognition and management of NMS and MC in a complex, gravely ill patient, may be accomplished in the ICU despite obfuscation of traditional signs and symptoms of the NMS and MC syndrome. Such interventions can have life-saving effects on patients in danger of fatal autonomic instability.
\end{abstract}

Keywords: neuroleptic malignant syndrome, malignant catatonia, neuropsychiatric syndrome, benzodiazepines

Received: 2 May 2020 / Accepted: 27 June 2020

\section{INTRODUCTION}

Neuroleptic Malignant Syndrome (NMS) is a neuropsychiatric syndrome characterized by hyperthermia, generalized "lead pipe" rigidity, elevation of creatine kinase, changes in mental status, and autonomic instability [1]. Dangerous consequences of NMS can result as the patient's condition progresses to rhabdomyolysis, hypertensive crisis, and metabolic acidosis. Treatment requires the discontinuation of the offending neuroleptic and aggressive hydration to protect the kidneys. Dantrolene (Par Pharmaceuticals, Chestnut Ridge, USA) can be used as a muscle relaxant and bromocriptine (Novartis, Basel, Switzerland) has shown effectiveness in potentiating dopamine pathways [2]. Across hospitals, NMS is an increasingly common occurrence in intensive care units (ICU) due to the regular use of antipsychotics for treatment of delirium [2].

However, the diagnosis of NMS can be confounded by similarities to conditions such as serotonin syndrome or catatonia and the inherent limitations of the physical and psychiatric exam in a critically ill, cachectic ICU patient, in a sedated state.

Catatonia is another complicated neuropsychiatric condition featuring significant psychomotor disturbances ranging from unresponsiveness or immobility to significant agitation and peculiar behaviors. Its diagnosis is made by satisfying three of the twelve criteria [1] (Table 1) and the degree of its clinical abnormalities can be rated using a catatonia rating scale such as the Bush-Francis Catatonia Rating Scale [3].

Stuporous motor immobility may be present in severe cases, whereas catalepsy and waxy flexibility may be the defining features of a more moderate case. Additionally, a single patient may demonstrate a wide range of psychomotor disturbances, making it difficult for clinicians to diagnose and treat [1]. Catatonia is often observed comorbid with psychiatric conditions such as schizophrenia and bipolar disorder, but it is also seen in patients with Autism Spectrum Disorder or Anti$\mathrm{N}$-methyl-D-aspartate receptor (anti-NMDAR) encephalitis. Malignant catatonia, a form of the disease 
Table 1. Catatonia is diagnosed clinically if at least 3 of the following 12 conditions are present *(1)

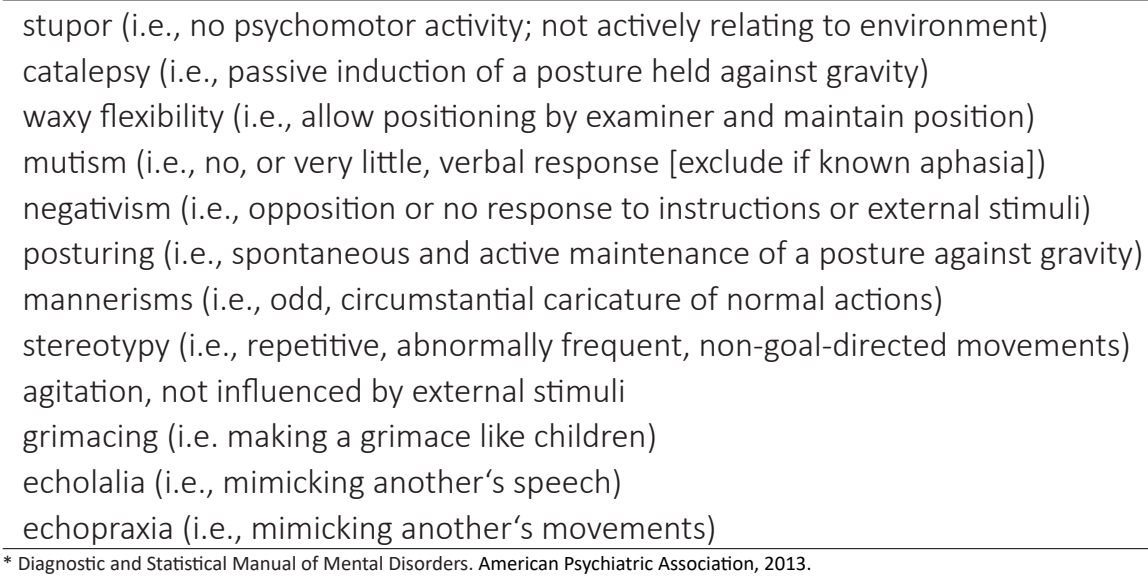

complicated by severe autonomic instability and fever is often life-threatening. However, evidence exists supporting the efficacy of benzodiazepines such as lorazepam (Pfizer, NY, USA) or electroconvulsive therapy in the treatment of malignant catatonia [4].

Recent literature has recognized many similarities in the presentation of NMS and catatonia and attempts have been made to reconcile the differences by suggesting that NMS and catatonia may represent different presentations of the same illness [4, 5]. Other studies have suggested that NMS and catatonia are distinct diseases but they lie along the same spectrum or are subtypes of a poorly understood clinical syndrome $[6,7]$. This commonality has led to the treatment of cases, that appear classically and are diagnosed as NMS, with benzodiazepines, which have also traditionally been used to treat catatonia [4].

A case of an ICU patient who presented with an difficult to diagnose case of NMS and catatonia and who responded to treatment with intravenous diazepam (Pfizer, NY, USA) is presented.

\section{- CAse Presentation}

The patient was a 22-year-old male who presented himself, without a referral, to an emergency center. This was his first presentation to healthcare, and he reported that one year prior to attending, he started experiencing chest discomfort and dyspnea on exertion. He had not visited or reported these issue to a general practitioner.

Upon arrival at the center, (Tertiary academic hospital, Philadelphia, PA) he developed cardiogenic shock due to right ventricular failure secondary to pulmo- nary hypertension. It was noted that he was cyanotic and hypoxic. Laboratory tests, taken post admission, showed an elevated white blood cell count, elevated liver function tests (LFT's) and moderate metabolic acidemia, lactic acid $5.7 \mathrm{mmol} / \mathrm{L}$, with normal hemoglobin, platelets, and electrolytes. Ultrasound showed right ventricular (RV) dysfunction with normal left ventricular (LV) function and a CT scan showed a possible right pulmonary embolus (PE).

He was then transferred as an emergency to the ICU.

On admission to the ICU he was intubated. While undergoing right heart catheterization, he had a vasovagal episode following puncture of his right internal jugular vein. His heart rate dropped from 80 beats per minute to 20 beats per minute, leading to a full cardiac arrest. He was resuscitated for approximately forty minutes and subsequently cannulated for venoarterial extra corporeal membrane oxygenation (VA ECMO).

Peri-arrest, his ejection fraction dropped to approximately $10 \%$. He was cooled and later regained neurological function. His CT imaging was reviewed and a pulmonary embolus (PE) was noted to be a motion artifact and the pulmonary hypertension was ruled as idiopathic.

Following development of a high fever three days later, colleagues in the Department of Infectious Disease were consulted. In spite of negative blood and broncho-alveolar lavage cultures, they recommended a seven day course of vancomycin (Pfizer, NY, USA) $1 \mathrm{~g}$ IV twice a day, plus ceftriaxone (Pfizer, NY, USA) $1 \mathrm{~g}$ IV daily, as a prophylactic against pneumonia.

After a two days of ceftriaxone the primary team decided to change this to meropenem, 2g IV daily (Pfizer, NY, USA) plus vancomycin, $1 \mathrm{~g}$ IV twice a day. 
Three days post-admission the patient was switched to receiving veno-venous extracorporeal membrane (VV ECMO) and de-cannulated.

Five days post-admission_bloody secretions were noted, secondary to pulmonary hemorrhage. Bronchoscopies were undertaken on more than one occasion for airway clearance. He was subsequently tracheostomized two weeks post-admission.

While in the ICU, the patient continued to spike fevers (Tmax 105F) in addition to developing severe nocturnal agitations with intermittent depressed responsiveness and somnolence. At that point the patient was prescribed quetiapine (AstraZeneca, Cambridge, UK) $50 \mathrm{mg}$ qhs, by feeding tube due to his agitation, in addition to fentanyl (Akorn, Lake Forest, USA) $1 \mathrm{mcg} / \mathrm{min}$. At this time magnetic resonance imaging (MRI) scans were normal.

Seventeen days post-admission, computerized tomography of chest, abdomen and pelvis showed multifocal lung opacities. A differential diagnoses of pneumonia or acute respiratory distress syndrome (ARDS) was suspected. A repeat bronchoscopy was performed but because there were no secretions, pneumonia was considered unlikely, especially after a seven-day, full course of vancomycin,ceftriaxone and meropenem.

Three weeks post-admission, due to agitation and intermittent somnolence, the constultation-liasion psychiatry team was consulted. The consultants agreed that the most likely cause of the temperature was a drug induced fever.

The psychiatry consultants referred to a case published by Mishima et. al (2011) which described how a patient developed NMS and malignant catatonia after a similar hospital course to the one prescribed in the current case [8].

However, in the current case, the diagnostic formulation was complicated by the fact that the patient was unable to speak due to his tracheostomy collar and was severely cachectic with critical illness myopathy. These masked the classic signs of rigidity and elevated creatine phosphokinase (CPK) seen in NMS. Furthermore, the typical signs of catatonia were difficult to elucidate due to the patient's mutism, weakness and intermittent sedation and fevers to $105^{\circ} \mathrm{F}$ had a very broad differential.

In spite of this, the agitation, diaphoresis, gegenhalten and recent use of neuroleptics, delirium pointed to a likely picture of NMS or malignant catatonia. The decision was made to discontinue quetiapine (Astra
Zeneca, Cambridge, UK) and start the patient on intravenous diazepam (Pfizer, NY, USA) 4mg IV qid.

Within twenty-four hours of starting diazepam the patient's fever, diaphoresis, white blood count, rigidity and alertness normalized. His family began to notice improvement in his level of alertness and a decrease in his overall agitation. The fentanyl was tapered off and the additional reassurance provided by the ICU team helped resolve the patient's agitation almost entirely. Careful observation indicated that the patient had no opioid withdrawal symptoms despite nine days of fentanyl, $1 \mathrm{mcg} / \mathrm{min}$. His temperature normalized, his condition gradually improved.

He was maintained on diazepam, 4mg IV qid, until his overall condition stabilized. His ejection fraction eventually improved to $75 \%$ and he responded well to the vasodilator sildenafil (Pfizer, NY, USA) $15 \mathrm{mg}$ IV tid, prescribed to treat pulmonary hypertension. Some residual contractures were noted on his upper extremities and his severe deconditioning was treated with physical and occupational therapy until he was discharged home on his current medical therapy.

\section{DISCUSSION AND CONCLUSIONS:}

Neuropsychiatric conditions such as NMS and catatonia are known to complicate the diagnosis and management of patients in the intensive care setting. NMS is a psychiatric syndrome thought to be caused by dopamine antagonism and is characterized by hyperthermia, "lead pipe" rigidity, elevated creatine kinase, delirium, and autonomic instability. Catatonia is characterized by psychomotor disturbances that may range from diminished responsiveness to marked agitation and may wax and wane daily in an individual.

NMS is a challenging diagnosis to make in the ICU setting due to a variety of confounding factors. In our patient's case, his cachexia made him incapable of mounting the traditional "lead pipe" rigidity and elevated CPK, despite the near-uniform presentation of those signs in the majority of patients with NMS. Because it was determined that the patient was uninfected, additional markers such as leukocytosis were used to make the diagnosis of NMS and treat appropriately. The lowering white blood count helped determine when discontinuation of diazepam could be undertaken. 
Lastly, in the context of NMS and malignant catatonia, intravenous diazepam proved to be a very effective treatment.

Malignant catatonia and NMS can be difficult to differentiate, especially considering the different subtypes of catatonia that can mimic or are believed to exist in the same spectrum as NMS [5, 9-11]. In this patient, distinguishing amongst the two proved to be extremely difficult because, the NMS' classic signs of rigidity and elevated CPK were disguised by the patient's severe weakness and cachexia. Moreover, mutism and negativism of malignant catatonia was difficult to elucidate due to his being non-verbal. Because fever had such a broad differential, it did not serve to distinguish malignant catatonia from NMS.

The key in this case was to closely consider that the etiology of his ongoing fevers to $105^{\circ} \mathrm{F}$, in spite of receiving prolonged broad spectrum antibiotics, was due to the recent use of neuroleptics, combined with recognizing that the patient's weakness masked the classic neuromuscular signs of rigidity seen in NMS, as well as masking the negativism and mutism associated with catatonia. Due to the increasing recognition that both conditions exist within the same spectrum, the decision was made to treat by discontinuing all neuroleptics and to provide diazepam instead of lorazepam because of literature suggesting its benefits [5], and because of a case report demonstrating its effectiveness in another patient who did not respond to an initial trial of lorazepam for a similar condition to our patient's [6].

\section{CONFLICT OF INTEREST}

None to declare.

\section{REFERENCES}

1. American Psychiatric Association: Diagnostic and Statistical Manual of Mental Disorders. Fifth Edition. Washington, DC, American Psychiatric Association, 2013.

2. Bienvenu OJ, Neufeld KJ, Needham DM: Treatment of four psychiatric emergencies in the intensive care unit. Crit Care Med 2012; 40(9): 2662-70.

3. Bush G, Fink M, Petrides G, Dowling F, Francis A: Catatonia. I. Rating scale and standardized examination. Acta Psychiatr Scand. 1996 Feb;93(2):129-36.

4. Sienaert $P, D$ hossche $D M$, Vancampfort $D$, et al: A clinical review of the treatment of catatonia. Front Psychiatry. 2014;5:181.

5. Lee JW:Neuroleptic-induced catatonia: clinical presentation, response to benzodiazepines, and relationship to neuroleptic malignant syndrome. J Clin Psychopharmacol. 2010 Feb;30(1):3-10

6. Vesperini S, Papetti F, Pringuey D. Existe-t-il un lien entre catatonie et syndrome malin des neuroleptiques ? [Are catatonia and neuroleptic malignant syndrome related conditions?]. Encephale. 2010;36(2):105-110.

7. Asztalos Z, Egervári L, Andrássy G, Faludi G, Frecska E. Katatónia és neuroleptikus malignus szindróma a pszichopatológiai és patofiziológiai átfedések tükrében: rövid összefoglaló tanulmány [Catatonia and neuroleptic malignant syndrome in view of a psychopathological and pathophysiological overlap: a brief review]. Neuropsychopharmacol Hung. 2014;16(1):1928.

8. Mishima T, Tsuboi Y, Higuchi MA, et al: Diazepam-responsive Malignant Catatonia in a Patient with an Initial Clinical Diagnosis of Neuroleptic Malignant Syndrome: A Case Report. Brain Nerve. 2011 May;63(5):503-7.

9. Lee, Joseph W. Y: Catatonic Variants, Hyperthermic Extrapyramidal Reactions, and Subtypes of Neuroleptic Malignant Syndrome. Ann Clin Psychiatry.2007 JanMar;19(1):9-16

10. Luchini F, Lattanzi L, Bartolommei N, et al: Catatonia and neuroleptic malignant syndrome: two disorders on a same spectrum? Four case reports. J Nerv Ment Dis. 2013 Jan;201(1):36-42

11. Roberto A, Coelho S, Sales P, et al: Malignant catatonia and neuroleptic malignant syndrome: two sides of the same coin? Eur Psychiatry. 2014; 29(1) 1 